\title{
Assessment of Functional Efficiency of Autonomic Nervous System in SCI Subjects Using RR Variability
}

\author{
A. G. Patil, R. H. Chile, and S. T. Hamde
}

\begin{abstract}
Although, good number of advanced modalities is available for diagnosis, there is no device that can measure functional efficiency of autonomic nervous systems in spinal cord injured subjects. There has been tremendous increase in number of spinal cord injured (SCI) patients around the world due to variety of reasons. The treatment of the spinal cord injury patient is mainly related to physical activity like: exercise, standing practice, balance control, etc. Cardiovascular health is most important issue for SCI patients today. Considering this, an attempt has been made to bring out a relation between percentage functioning of autonomic nervous systems and RR variability. Efforts have been made to define the efficiency of the spinal cord or ANS on the basis of components $s, p$, and $n$. They represent functioning of sympathetic, parasympathetic and ANS respectively. Component values are in percentage between 0 and 100 . It is observed that the suggested model efficiently presents status of autonomic nervous system and the impact of injury on the spinal cord.
\end{abstract}

Index Terms-Functional efficiency in SCI, RR variability, Spinal cord injury.

\section{INTRODUCTION}

The vertebral column of human body is classically divided into various levels: the 7 cervical vertebrae, 12 thoracic, 5 lumbar, 5 sacral and 4 coccygeal. Within the vertebral column, the spinal cord is protected ventrally by the bony laminae, and laterally by the pedicles. Spinal cord is a cylinder of nerve tissue about the thickness of little finger and has a length of about 38 to 45 centimeters. It joins the medulla oblongata in the brain and runs down to a level of first lumber vertebra where it ends into a bunch of nerves called cauda equine [1]. The nervous system is the main communication system between various parts of the body. Functioning of the nervous system is based on generation of bio-potentials and their propagation. The autonomic nervous system supplies nerves to all the internal organs of human body including blood vessels. It is called autonomic because the organs under this system are self-controlled and not under the control of individual's will. The autonomic nervous system has two parts: sympathetic nervous system and parasympathetic nervous system. These two systems have in each case, opposite actions. One is stimulating and another is checking or inhibiting.

Spinal cord injury can occur anywhere on the spinal cord.

Manuscript received June 5, 2012; revised July 9, 2012.

A. G. Patil is with the Shri Bhagubhai Mafatlal Polytechnic, Vile Parle (West), Mumbai, India (tel.: + 91-22-26207175, +91-22-42336004; fax: +91-22-26110117; e-mail: agpatil@ rediffmail.com).

R. H. Chile and S. T. Hamde are with the Department of Instrumentation, S.G.G.S. Institute of Engineering and Technology Vishnupuri, Nanded, India.
Injuries at the thoracic level and below result in paraplegia Paraplegia describes complete or incomplete paralysis that affects legs and possibly also the trunk, but not the arms. Cervical or neck injuries usually result in four-limb paralysis. This is sometimes referred to as Tetraplegia or Quadriplegia [2]-[3]. Destruction of a small portion of the spinal cord produces profound motor and sensory changes below the level of the injury. Spinal Cord Injury may show symptoms and signs that the autonomic system has been affected by the injury. Studies of cardiovascular abnormalities after spinal cord injury show that of subjects with severe cervical injuries develop bradycardia, hypotension, and some of them suffer from primary cardiac arrest [2]-[7].

Considering increasing number of spinal cord injured (SCI) patients and need for properly guided rehabilitation, there is no mechanism or modality available that can easily define cardiovascular health of the patient for further treatment. The treatment of SCI patient is mainly related to physical activity like: exercise, standing practice, balance control, etc. Cardiovascular health is most important issue for SCI patients today, and ECG alone can't certify it [8]-[9]. The electromyography and evoked response systems can provide a selective data related to nerve conduction and evoked responses. But, it cannot analyze overall response of autonomic nervous system. Imaging modalities like: X-Ray, CT-Scan, MRI, Ultrasonography, etc, provide only structural information i.e. physical damage. This is not sufficient to determine functional efficiency or damage to the system and therefore to forecast patient's future. The general theme of rehabilitation in the spinal cord patient is to make maximum use of the remaining function and achieve highest degree of independence. Therefore, RR variability analysis would emerge as an important tool for assessment of spinal cord injury and cardiovascular health. Application of this tool would also help the society in general to assess the cardiovascular health. It would also help to detect in advance unknown or known disease in early stage. The R-R variability is a non-invasive index of the neural control of the heart. In R-R variability analysis the intervals between successive QRS complexes need to be determined. There are two methods of analysis of R-R variability data: time- and frequency-domain analysis. In either method, the inter-beat intervals should be properly calculated and all abnormal heartbeats and artifacts removed from consideration [2]-[7].

\section{MATERIALS AND Methods}

A cardiosport belt based wireless system has been developed and used for this work to acquire RR intervals [8]-[13]. Spinal cord injured subjects can't move or perform 
activities that are required to obtain response of the heart. Therefore, it was decided to record the response during supine, sitting and deep breathing modes of positions. Two subject groups have been studied in present work. They are: spinal cord injured patients and healthy normal subjects. The first group consists of 38 healthy persons of 17 to 60 years old and second group has 20 persons with spinal cord injury. In SCI group 10 subjects have cervical injury, 8 have thoracic injury and remaining 2 have lumber level spinal cord injury. All normal subjects were healthy and none of them was suffering from any known disease at the time of recording [2]-[9].

In this study, RR intervals are recorded in three different modes or positions: supine, sitting and five seconds deep respiration. The RR interval data of 150 seconds in each mode found sufficient to produce subject's response. Three new indices: DOS, DRS and ROR are defined [2]-[9]. These indices reveal subject's response to orthostatic and respiratory stress very efficiently. These indices play major role in assessing functional activity of nervous system.

1) DOS: (Dynamic Orthostatic Stress Index): The difference between sympathetic and para-sympathetic balance indices during sitting and supine referred to supine mode.

$$
D O S=\frac{S t B-S u B}{S u B}
$$

2) DRS: (Dynamic Respiratory Stress Index): The difference between inverse of sympathetic and para-sympathetic balance indices during deep breathing and supine referred to supine mode.

$$
D R S=\frac{(1 / D b B)-(1 / S u B)}{(1 / S u B)}
$$

3) ROR: (Ortho-Respiratory Stress Index): Ratio of Balance during Sitting to Balance during Deep Respiration.

$$
R O R=\frac{S t B}{D b B}
$$

where:

SuB: Sympathetic to Para-Sympathetic Balance Index Supine

Ratio of spectral power in the low frequency range $(0.04-$ $0.15 \mathrm{~Hz}$ ) to the spectral power in the high frequency range $(0.15-0.4 \mathrm{~Hz})$ during supine mode.

$$
\mathrm{SuB}=\operatorname{SPL}(\mathrm{Su}) / \mathrm{SPH}(\mathrm{Su})
$$

StB: Sympathetic to Para-Sympathetic Balance Index Sitting

Ratio of spectral power in the low frequency range $(0.04-$ $0.15 \mathrm{~Hz})$ to the spectral power in the high frequency range (0.15 - $0.4 \mathrm{~Hz}$ ) during sitting mode.

$$
\mathrm{StB}=\mathrm{SPL}(\mathrm{St}) / \mathrm{SPH}(\mathrm{St})
$$

$D b B$ : Sympathetic to Para-Sympathetic Balance IndexDeep Breathing

Ratio of spectral power in the low frequency range $(0.04-$
$0.15 \mathrm{~Hz})$ to the spectral power in the high frequency range (0.15- $0.4 \mathrm{~Hz}$ ) during deep respiration mode.

$$
\mathrm{DbB}=\mathrm{SPL}(\mathrm{Db}) / \mathrm{SPH}(\mathrm{Db})
$$

\section{A. Assessment of Functional Efficiency}

To assess the functional efficiency, the model representing functional efficiency of sympathetic system, para-sympathetic system and autonomic nervous system based on indices derived from RR variability has been developed [9], and presented. The model is shown in fig. 1. It consists of components $s, p$, and $n$. The component ' $s$ ' represents the functioning of sympathetic system, component ' $p$ ' represents functioning of parasympathetic system and ' $n$ ' indicates functioning of ANS or overall system. Component values are in percentage between 0 and 100 .

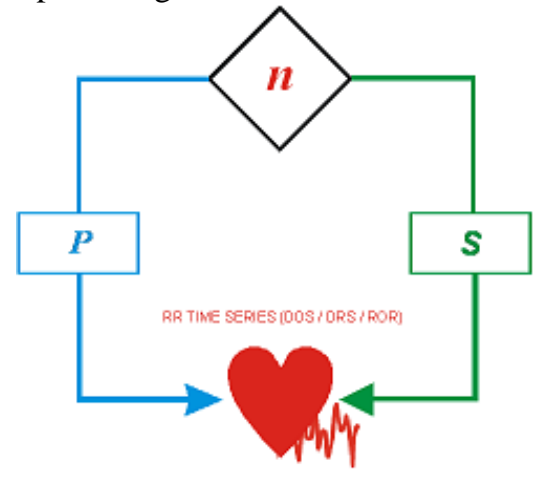

Fig. 1. Model of RRV

\section{B. Model Parameters}

The DOS, DRS and ROR indices of 38 normal subjects are referred to gather base values of $s, p$ and $n$. DOS, DRS and ROR represent complete picture of RR variability that depends on functioning of sympathetic and parasympathetic activity. The relation between DOS, DRS and ROR indices for normal and spinal cord injured subjects is shown in fig. 2 using bar and radar graphs. DOS index draws maximum response from sympathetic activity due to orthostatic stress, while DRS produces extreme response from parasympathetic activity when the subject is engaged in deep respiration. Therefore, the index ROR, which is result of these two activities, represents status of autonomic nervous system [9]. In these graphs, DOS index shows significant reduction from 2.19 in normal subjects to 0.62 in SCIs. This is a clear indication of damage to the sympathetic and parasympathetic systems. A similar trend is indicated in DRS index showing reduction in index value. This index gives the effectiveness of vagal control on RR interval or heart rate and is considerably reduced from 4.76 for normal subjects to 0.17 in SCI subjects. Thus, the Ratio of Balance during Sitting to Balance during Deep Respiration (ROR) is also severally affected for SCIs. For normal subjects it is 14.41, whereas for SCIs it is only 3.2. Graphical images of all these indices present the typical variability picture of SCI patients, which is significantly different from normal subjects. The radar graph representing these indices clearly shows two different triangles not meeting at any corner. Not only this, but highly restricted activity in small area at the center of graph for SCIs in contrast to normal subjects. 

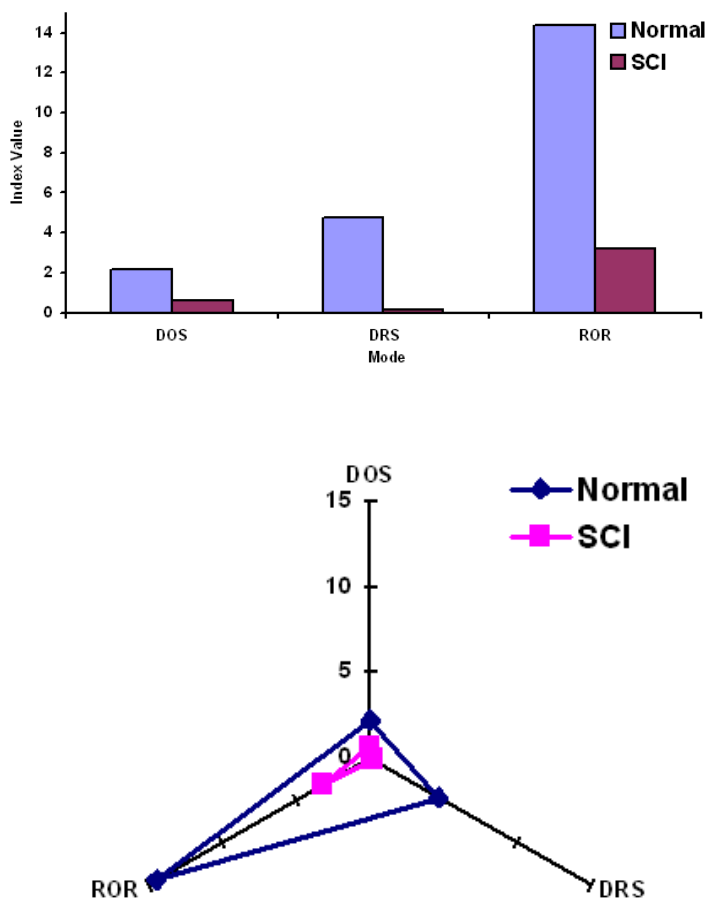

Fig. 2. Relation between DOS, DRS and ROR.

Distributions of DOS, DRS and ROR indices were produced using DADiSP [14] software to observe the distribution of index values. Distribution and peak point of maximum distribution was then derived by taking moving average. Distribution of DOS index is shown in fig. 3. It shows that the distribution is concentrated at index value ' 2 '. Therefore, this value is considered as a base value for this parameter. The DOS value of ' 2 ' is equivalent to $100 \%$ of sympathetic function or parameter ' $s$ ' [9].

Distribution of DRS index is shown in fig. 4. The graph reveals distribution of index value at ' 4 '. Hence, this is considered as a base value for DRS. The DRS value of ' 4 ' is equivalent to $100 \%$ parasympathetic function or parameter ' $p$ '. Distribution of ROR index is shown in fig. 5. The graph brings out distribution of index value as ' 12 ' and this is considered as a base value for ROR. This ROR value is equivalent to $100 \%$ total anatomic function or parameter ' $n$ '. These derived base values for three functions are considered as RR model parameters [9].

As discussed above the relationship of parameter 's', ' $p$ ' and ' $n$ ' are: $s \propto$ DOS, $p \propto$ DRS and $n \propto$ ROR.

Therefore,

1) Percentage functional ability of sympathetic system is =

$$
s=\frac{D O S}{2} \times 100
$$

2) Percentage functional ability of parasympathetic system is $=$

$$
p=\frac{D R S}{4} \times 100
$$

3) Percentage functional ability of autonomic nervous system is $=$

$$
n=\frac{R O R}{12} \times 100
$$

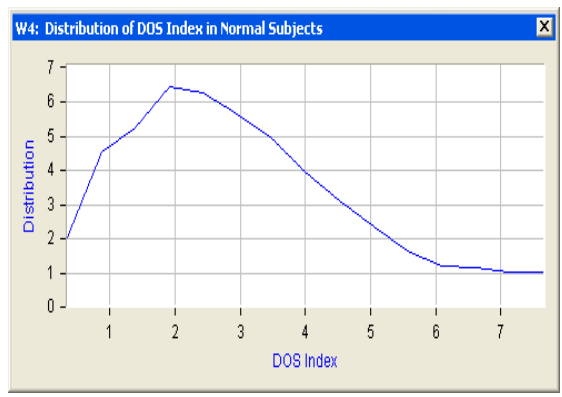

Fig. 3. DOS index.

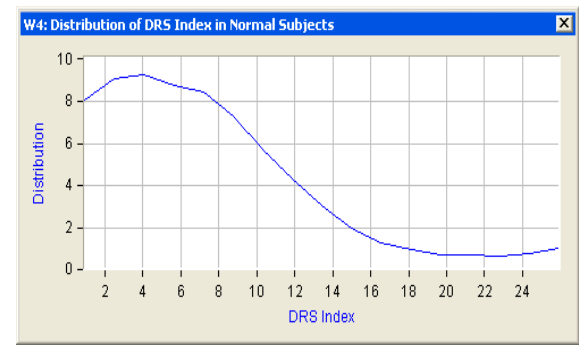

Fig. 4. DRS index.

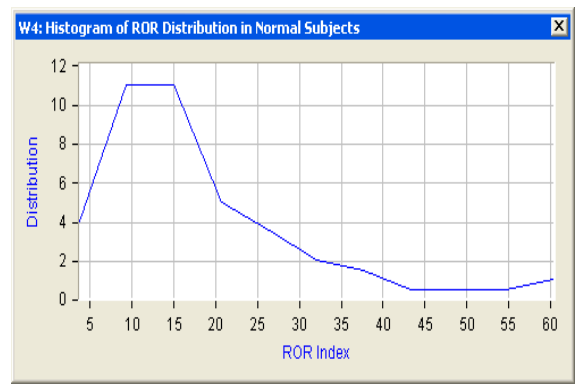

Fig. 5. ROR index.

All parameters are in the range 0 to 100 . Values above 100 and below 0 are converted in to 100 and 0 respectively.

\section{Results and Discussion}

By referring the data generated using expressions stated above, functional efficiencies of sympathetic, parasympathetic and autonomic nervous systems are calculated. Findings are presented in Table I. These findings are correlated with individual subject having spinal cord injury. Here, subject's injury level and physical assessment as per International Spinal Cord Injury Core Data Set [14] (ASIA) are presented along with model parameters. It shows functioning of sympathetic, parasympathetic and autonomic nerves system i.e. status of spinal cord in spinal cord injured patients.

In this case none of the patient shows total function above $55 \%$. Here, 4 subjects have functional ability between 40-55 \%, 9 have between 20-40\% and remaining 7 have below $20 \%$. Sympathetic functional ability in 2 subjects is $100 \%$, in 1 subject it is between $72.6 \%$, in 5 subjects it is between $20-40 \%$ and in 12 it is below $20 \%$. Surprisingly, parasympathetic functional ability is below $20 \%$ in all the subjects. 
TABLE I: FUNCTION DETAILS OF SCI SUBJECTS.

\begin{tabular}{|c|c|c|c|c|c|}
\hline & $s(\%)$ & $p(\%)$ & $n(\%)$ & Injury (ASIA) & Physical assessment \\
\hline$S-1$ & 7.8 & 2.3 & 29.0 & Cervical (D) & Above $\mathrm{C} 4$, numbness in whole body, can walk, very weak, limited activity \\
\hline$S-2$ & 100 & 8.1 & 45.9 & Cervical (C) & $\begin{array}{l}\text { C4-C5, severe injury, incomplete sensations, little movements, wheel } \\
\text { chair confined, very active }\end{array}$ \\
\hline$S-3$ & 11.3 & 2.1 & 18.3 & Thoracic (B) & $\begin{array}{l}\text { T3, T4, severe injury, incomplete sensations, no movements, wheelchair } \\
\text { confined, no activity }\end{array}$ \\
\hline$S-4$ & 3.2 & 2.1 & 26.1 & Thoracic (C) & $\begin{array}{l}\text { T1-T4, severe injury, incomplete sensations, little movements, wheelchair } \\
\text { confined, limited activity }\end{array}$ \\
\hline$S-5$ & 0.7 & 0.5 & 9.2 & Cervical (A) & $\begin{array}{l}\text { C4-C6, severe injury, no movements, no sensations below neck, bed } \\
\text { ridden, no activity }\end{array}$ \\
\hline$S-6$ & 9.7 & 1.4 & 23.2 & Cervical (D) & C4-C7, good recovery, can walk with support, limited activity \\
\hline$S-7$ & 36.1 & 1.9 & 16.4 & Thoracic (B) & $\begin{array}{l}\text { T1, incomplete sensations, no movement, national champion, wheelchair } \\
\text { confined, limited activity }\end{array}$ \\
\hline$S-8$ & 23.7 & 1.1 & 16.8 & Cervical (B) & $\begin{array}{l}\text { C5-C6, severe injury, no movements, slight sensations, wheelchair } \\
\text { confined, limited activity }\end{array}$ \\
\hline$S-9$ & 43.5 & 13.6 & 49.2 & Thoracic (C) & $\begin{array}{l}\text { T11, can stand with splint, incomplete sensations, little movement, daily } \\
\text { exercise, very active }\end{array}$ \\
\hline$S-10$ & 23.1 & 17.8 & 41.5 & Cervical (C) & $\begin{array}{l}\text { C3, can stand and walk with support, incomplete sensations, daily } \\
\text { exercise, very active }\end{array}$ \\
\hline$S-11$ & 16.7 & 0.5 & 22.2 & Thoracic (B) & $\begin{array}{l}\text { T5-T6, incomplete sensations, little movements, wheelchair confined, } \\
\text { limited activity, active }\end{array}$ \\
\hline$S-12$ & 43.7 & 1.6 & 24.2 & Cervical (B) & $\begin{array}{l}\text { C1-C4-L1, serious injury, incomplete sensations, no movements, } \\
\text { wheelchair confined, very active }\end{array}$ \\
\hline$S-13$ & 25.5 & 1.7 & 21.5 & Thoracic (B) & $\begin{array}{l}\text { T4-T5, serious injury, incomplete sensations, no movements, wheelchair } \\
\text { confined, active }\end{array}$ \\
\hline$S-14$ & 100 & 8.1 & 54.4 & Cervical (D) & Cervical Spondylosis, can walk, very active \\
\hline$S-15$ & 5.7 & 16.3 & 38.4 & Cervical (D) & Cervical Spondylosis, can walk, very active \\
\hline$S-16$ & 24.2 & 2.1 & 33.6 & Lumbar (D) & Cervical Spondylosis, can walk, very active \\
\hline$S-17$ & 72.6 & 5.1 & 33.1 & Thoracic (C) & $\begin{array}{l}\text { L1-L5, incomplete sensations, little movements, wheelchair confined, } \\
\text { very active }\end{array}$ \\
\hline$S-18$ & 5.2 & -0.8 & 8.1 & Cervical (A) & $\begin{array}{l}\text { SCI by birth, C3-C6, no sensations, no movements, wheelchair confined / } \\
\text { bed ridden, no activity }\end{array}$ \\
\hline$S-19$ & 29.6 & -0.4 & 11.4 & Thoracic (A) & $\begin{array}{l}\text { T5-T6, no sensations, no movements, new bladder implanted, wheelchair } \\
\text { confined, artist, active }\end{array}$ \\
\hline$S-20$ & 18.9 & 0.2 & 12.9 & Lumber (B) & L2-15, incomplete sensations, no movement, wheelchair confined, active \\
\hline
\end{tabular}

\section{CONCLUSION}

This study establishes the relation between functional efficiency of autonomic nervous system and RR variability. Many studies have been published during recent years indicating a strong association between autonomic balance and mortality, with a deficiency in vagal tone a primary marker of increased risk. Sympathetic and parasympathetic functions are independent functions like push-pull mechanism. Therefore, effectiveness of autonomic nerves system or the natural control mechanism depends on their balancing act. If one function is very strong and the other 
very weak, there is no effective control. Hence, total efficiency of autonomous nervous system $(n)$ can't be derived only from ' $s$ ' and ' $p$ ', but by assessing it separately. It has been observed from these findings that even if the values of ' $s$ ' and ' $p$ ' are small, but slightly balanced, overall efficiency $(n)$ is better and is useful. The results show that the model fits to the physical condition of the SCI subject. Subjects, who are active or engaged in daily exercise, clearly show improvement in functioning of autonomous nervous system. But, in this case, there is much improvement in sympathetic function compared to parasympathetic function. It was assumed that level of injury must be playing important role in deciding the functional activity. But, it seems that level of injury doesn't matter, but the depth of injury matters. Lower level injuries i.e. thoracic and lumber also affect the function same way as higher level i.e. cervical injuries. Spondylosis affects both the functions, but overall function is moderately affected. It is observed that the suggested model efficiently presents status of autonomic nervous system and therefore spinal cord. As the group undertaken for study has limited number of subjects, the results obtained should be taken in right spirit for further research. The detailed correlation between normal and spinal cord injured subjects need to be established by applying this procedure to larger group.

\section{REFERENCES}

[1] D. C. Burke and D. D. Murry, Handbook of Spinal Cord Medicine, The Macmillan Press Limited, London, 1975.

[2] A. G. Patil, R. H. Chile, and S. T. Hamde, "RR Variability in Spinal Cord Injured Patients," The Internet Journal of Bioengineering. vol. 3 no. 1,2008 ,

[3] A. G. Patil, R. H. Chile, and S. T. Hamde, "Spinal cord injury alters RR variability: a comparative studybetween normal and spinal cord injured persons," Proceedings of WACBE World Congress on Bioengineering, Bangkok, Thailand, July 2007.

[4] A. G. Patil, R. H. Chile, and S. T. Hamde, "System for study and analysis of R-R time Series signal in spinal cord injury patients," Proceedings of BIOCON-2005-National Conference on Emerging Trends in Biomedical Engineering, Pune, India, 2005.

[5] M. Malik (Chairman), "Heart rate variability, standards of measurement, physiological interpretation, and clinical use, task force of the European society of cardiology the north American society of pacing electrophysiology," Circulation, 1996, vol. 93, pp. 1043-1065

[6] J. N. Myers, K. Gladysheva, BA, H. E. Brown, I. Perkash, and B. Jenny Kiratli, "Heart rate variability and physical activity level after spinal cord injury," VA Rehabilitation Research and Development Service, project \#B2549-R

[7] J. M. Legramante, G. Raimondi, M. Massaro, and F. Iellamo, "Positive and negative feedback mechanisms in the neural regulation of cardiovascular function in healthy and spinal cord-injured humans," Circulation, 2001, vol. 103, pp. 1250-1255.

[8] M. DeVivo, F. Biering-Sørensen, S. Charlifue, V. Noonan, M. Post, T. Stripling, and P. Wing, "Executive committee for the international SCI data sets committees," International Spinal Cord Injury Core Data Set. Spinal Cord. Sep, 2006, vol. 44, no. 9, pp. 535-40.

[9] A. G. Patil, R. H. Chile, and S. T. Hamde, "Statistical Analysis of RR Series Variability in Spinal Cord Injured Persons," Journal of Medical Engineering and Technology, informa healthcar, 2012, vol. 36, no. 3, pp. $180-184$.

[10] A. G. Patil, R. H. Chile, and S. T. Hamde, "Model based analysis of sympathetic and parasympathetic nervous systems in spinal cord injured subjects using rr variability," in proc of 2012 2nd International Conference on Biomedical Engineering and Technology, IPCBEE vol. 34, Hong Kong 2012.

[11] A. A. Patil and A. G. Patil, "Wireless R-R interval data acquisition and analysis for study of heart rate variability," in Proceedings of WACBE World Congress on Bioengineering, Bangkok, Thailand, July 2007.

[12] A. G. Patil, R. H. Chile, and S. T. Hamde, "Wireless system for measurements of rr variability during physical activity," The Internet Journal of Bioengineering, vol. 3, no. 2, 2008.

[13] Cardiosport, Cardiosport Chest transmitter [Online]. Available: http:// www.heartratemonitor.co.uk

[14] DADiSP, Digital Signal Processing Software, DSP Development Corporation. [Online]. Available: http:// www.dadisp.com. 